\title{
Level-1 trigger selection of electrons and photons with CMS for LHC Run-II
}

Thomas STREBLER*广

Ecole Polytechnique / CNRS-IN2P3, Palaiseau, France

E-mail: thomas.streblerallr.in2p3.fr

The CMS experiment has a sophisticated two-level online selection system that achieves a rejection factor of nearly $10^{5}$. The first, hardware-level trigger (L1) is based on coarse information coming from the calorimeters and the muon detectors while the High-Level Trigger combines fine-grain information from all subdetectors. During Run II, the LHC will increase its center of mass energy to 13 or $14 \mathrm{TeV}$, and progressively reach an instantaneous luminosity of $2 \times 10^{34} \mathrm{~cm}^{-2} \mathrm{~s}^{-1}$. In order to guarantee a successful and ambitious physics programme in this intense environment, the CMS trigger and data acquisition system must be upgraded. The L1 calorimeter trigger hardware and architecture in particular has been redesigned to maintain the current thresholds even in presence of more demanding conditions (e.g., for electrons and photons) and improve the performance for the selection of $\tau$ leptons. This design benefits from recent $\mu$ TCA technology, allowing sophisticated algorithms to be deployed, better exploiting the calorimeter granularity and opening the possibility of making correlations between different parts of the detector. The electron/photon selection algorithm uses an innovative dynamic clustering procedure along with an online pile-up correction technique which represents a real challenge for an electronics trigger system. The performance of this electron/photon trigger will be demonstrated, both in terms of efficiency and rate reduction. The new features to control rates in different pile-up scenarios will be described. Finally, the plans for the commissioning with the first Run II data will be presented and the expected impact on the physics potential assessed.

XXVII International Symposium on Lepton Photon Interactions at High Energies 17-22 August 2015

Ljubljana, Slovenia

${ }^{*}$ Speaker.

${ }^{\dagger}$ On behalf of the CMS collaboration 


\section{Introduction}

During the Run I of the Large Hadron Collider (LHC), the CMS experiment [1] could rely on an efficient triggering of leptons and photons down to low transverse momenta (down to $20 \mathrm{GeV}$ for L1 single electron/photon trigger). The data taking conditions of the LHC Run II represent a real challenge for the hardware-level (L1) trigger system. Indeed, the increased center of mass energy to $\sqrt{s}=13 \mathrm{TeV}$ implies an overall physics rate increased by a factor of 4 , while the L1 output rate must be maintained below $100 \mathrm{kHz}$. The average number of interactions per bunch crossing (pile-up) is also increased from around 10 to around 50 interactions per crossing. In order to maintain low $p_{T}$ thresholds and mitigate the effect of pile-up, the CMS L1 trigger system had to be upgraded [2]. The new L1 selection of electrons and photons, based on the L1 calorimeter upgrade, is presented.

\section{Technological developments}

The upgrade of the CMS L1 trigger system largely benefits from hardware improvements, including new optical link boards and recent developments in $\mu \mathrm{TCA}$ electronic cards. Unlike the Run I calorimeter trigger, which was spatially segmented into separate processing elements, the upgraded trigger system can base its decision on the full detector information. This is possible thanks to a new Time-Multiplexed Trigger (TMT) architecture. This new architecture implements 36 Layer-1 cards (CTP7) that send data from the whole detector for a given bunch crossing to one of the 12 Layer-2 cards (MP7). Each of the MP7 boards has to deliver a decision to the Global Trigger within a 10 bunch crossings interval. Thanks to these developments, the L1 trigger algorithms for the selection of electrons and photons can be largely improved and based on sophisticated techniques.

\section{Dynamic clustering and calibration}

One of the new features of the trigger algorithms is the implementation of a dynamic clustering to reconstruct the energy deposit corresponding to electrons or photons in the calorimeter: trigger towers (groups of calorimeter cells used as trigger units) corresponding to local energy maxima are used as seed for the clusters and the neighboring energy deposits in a window of 3x5 trigger towers in $\eta-\phi$ are clustered around the seeds. In order to optimize the resource usage on the electronic boards, the clusters produced are also used to produce L1 $\tau$ candidates. This dynamic clustering allows to efficiently reconstruct the electrons with a large amount of Bremsstrahlung radiation. The energy distribution in the cluster is also used to compute a refined position of the electron/photon candidate with a better precision than the tower granularity. The position resolution is thus significantly enhanced with respect to the Run 1 algorithm, as can be seen in Fig 1.

The L1 electron/photon algorithm also includes an energy calibration of the candidates. Energy corrections are applied such that the transverse energy of the L1 trigger candidates corresponds to that of the offline reconstructed electrons. They depend on the uncalibrated energy of the cluster, on its position in $\eta$ and on its topological shape. The obtained energy resolution is presented in Fig. 2. Superior resolution can be achieved with respect to the Run 1 trigger since the upgrade benefits from the enhanced calorimeter granularity through the dynamic clustering. 

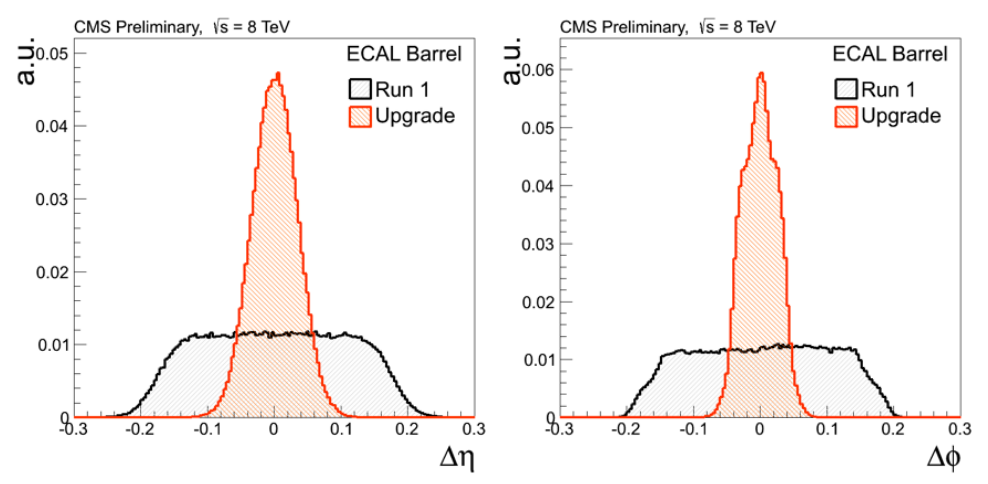

Figure 1: Position resolution of the $\mathrm{L} 1$ electron/photon candidates, evaluated in $8 \mathrm{TeV}$ data using tag-andprobe techniques. The resolution is computed with respect to offline-reconstructed electrons. [3]
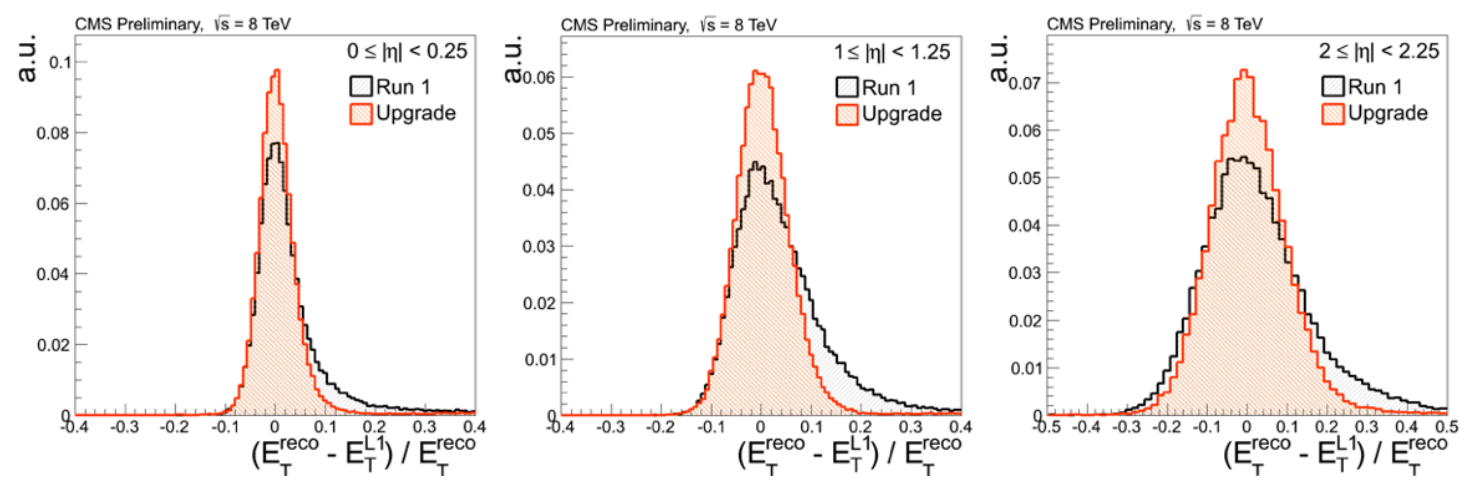

Figure 2: Transverse energy resolution of the $\mathrm{L} 1$ electron/photon candidates in different $\eta$ regions, evaluated in $8 \mathrm{TeV}$ data using tag-and-probe techniques. The resolution is computed with respect to offlinereconstructed electrons. [3]

\section{Background reduction}

In order to reduce the background rate, some identification criteria are applied on the candidates. This identification is based on the energy distribution within the seed trigger tower (called fine grain), the ratio of the energy deposit in the seed trigger tower between the electromagnetic and hadronic calorimeters and the shape of the cluster (which is typically smaller for electromagnetic objects than for jets). An isolation flag is also computed for each candidate, taking into account the level of pile-up, estimated from the number of trigger towers with a non-zero energy deposit in the central region of the calorimeter. A pile-up dependent cut is then applied on the energy in an isolation region around the clusters to separate isolated and non-isolated candidates.

In terms of efficiency, the upgraded trigger shows sharper turn-on curves than the Run 1 algorithm, as seen in Fig. 3 (left). This improvement is directly linked to its better energy resolution. The new trigger also achieves a rate reduction after isolation improved by a factor 2 , as seen in Fig. 3 (right). Along with the sharper turn-on curves, this improvement is due to the use of cluster shapes that are used to discriminate between electromagnetic objects and jets. 

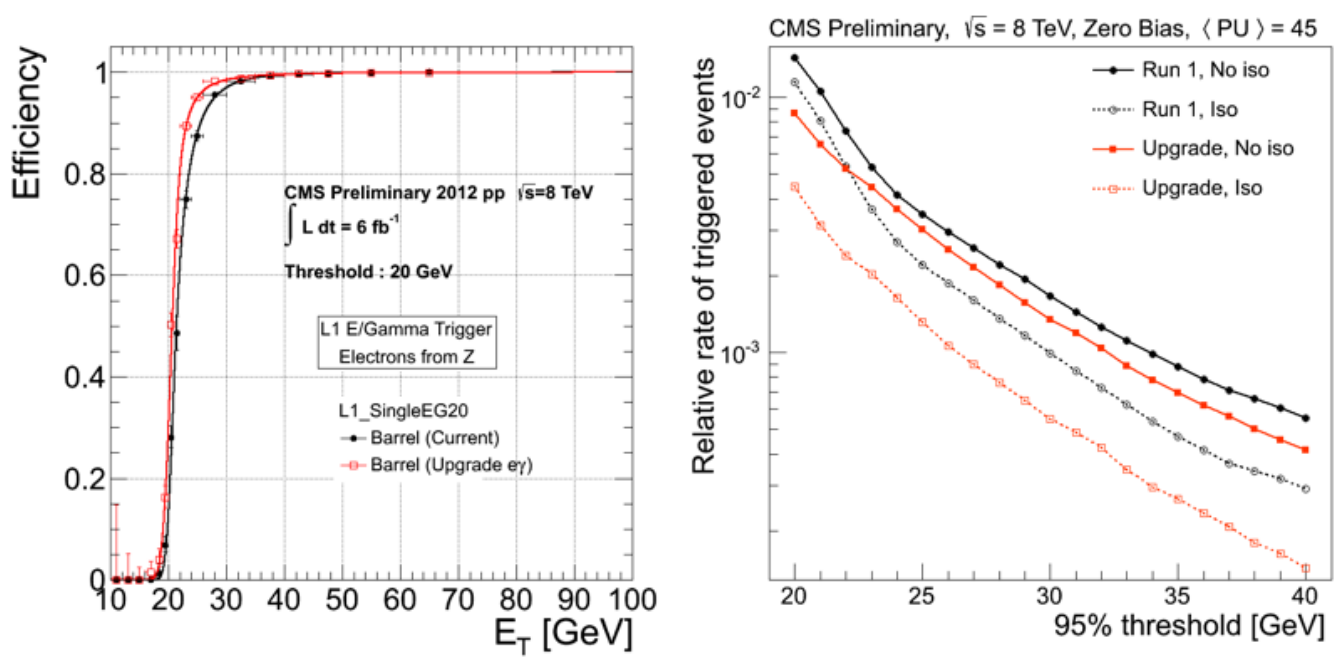

Figure 3: Turn-on curves in the barrel (left) and relative trigger rates as a function of the $95 \%$ efficiency threshold (corresponding to the offline $E_{T}$ where the trigger reaches $95 \%$ of its plateau efficiency) (right). The efficiency is plotted for a given trigger threshold of $20 \mathrm{GeV}$, as a function of the transverse energy of the offline-reconstructed electrons, using tag-and-probe selections with $8 \mathrm{TeV}$ data. The trigger rates are evaluated in $8 \mathrm{TeV}$ zero-bias data with high pile-up (average of 45 pile-up interactions per bunch crossing) [3].

\section{Conclusion}

Thanks to important technological developments, the L1 trigger of CMS for electrons and photons benefits from a significant upgrade which will enable to maintain high performance in the challenging conditions of Run II. The commissioning of the new trigger with data should start in September 2015, where it will run in parallel with the Run 1 algorithms, and the upgraded trigger will be used for physics from 2016 onwards.

\section{Acknowledgment}

The work of the author of this paper has been partly funded by the P2IO LabEx (ANR-10LABX-0038) in the framework "Investissements d'Avenir" (ANR-11-IDEX-0003-01) managed by the French National Research Agency (ANR).

\section{References}

[1] CMS Collaboration, The CMS Experiment at the CERN LHC, JINST 3 (2008) S08004

[2] CMS Collaboration, CMS technical design report for the Level-1 Trigger upgrade, CERN-LHCC-2013-011, CMS-TDR-12 (2013)

[3] CMS Collaboration, L1 calorimeter trigger upgrade: electron and photon performance, CMS-DP-2015-003 (2015) 